\title{
Chromium isotope composition of Apollo samples: implication for galactic cosmic ray induced reactions

\author{
PRÉCILLIA MORINO ${ }^{1}$, MARIA SCHÖNBÄCHLER ${ }^{2}$, \\ COLIN MADEN $^{2}$ AND HENNER BUSEMANN ${ }^{2}$
} \\ ${ }^{1}$ Institute of Geochemistry and Petrology, ETH Zürich, \\ ${ }^{2}$ ETH Zürich \\ Presenting Author: precillia.morino@erdw.ethz.ch
}

It is generally accepted that the Moon formed by a giant collision between proto-Earth and a large impactor, Theia [e.g., 1]. Nevertheless, the composition of Theia remains subject to debate. Several isotopic studies show strong similarities between bulk silicate Earth (BSE) and the Moon [e.g., 2], constraining the formation scenario. A recent $\mathrm{O}$ isotope study [3] proposes, however, that this similarity is only apparent and remnants of Theia could be preserved in the deep reservoirs of the Moon. Therefore, it is essential to continue to characterize its isotopic composition with high precision analyses. Each planetary body in our solar system, except Moon and Earth, has its own nucleosynthetic ${ }^{54} \mathrm{Cr}$ isotope compostion and thus ${ }^{54} \mathrm{Cr}$ data have the potential to further constrain the origin of Theia, when compared the solar system inventory [2]. To this end, ultraprecise $\mathrm{Cr}$ isotope data for lunar samples are required. Nuclear reactions induced by exposure to galactic cosmic rays (GCR) affect the $\mathrm{Cr}$ isotope composition of lunar samples and need correction to obtain the initial isotope compostion [4]. The production of cosmogenic $\mathrm{Cr}$ depends on several factors such as chemical composition of the rock, exposure parameters and nature of the GCR-induced reactions [4,5]. Since noble gases are extremely sensitive to GCR irradiation, this study combines He, $\mathrm{Ne}, \mathrm{Ar} \mathrm{Kr}$ and $\mathrm{Xe}$ analyses with ultra-high precision $\mathrm{Cr}$ isotope data ( $<5$ and $7 \mathrm{ppm}$ for $\varepsilon^{53} \mathrm{Cr}$ and $\varepsilon^{54} \mathrm{Cr}$ (2SE), respectively) for 18 Apollo samples. All isotope measurements are being carried out on the same sample aliquots at ETH Zürich. Our results show that $\mathrm{Cr}$ isotope data correlate with the duration of exposure and the specific GCR-induced reactions involved.

[1] Canup, R. M. (2004) Icarus 168:433-456. [2] Akram, W. \& Schönbächler, M. (2016) Earth Planet. Sci. Lett. 449:302-310. [3] Cano (2020). Nature Geoscience, 13:270-274 [4] Leya I. et al. (2003) Geochim. Cosmochim. Acta 67:529-541. [4] Mougel B. et al. (2018) Earth Planet. Sci. Lett. 481:1-8. [5] Liu J. et al. (2019) Geochim. Cosmochim. Acta 251:73-86. 